\title{
EXISTENCE OF SOLUTIONS TO WEAK NONLINEAR BILEVEL PROBLEMS VIA MINSUP AND D.C. PROBLEMS
}

\author{
AbDelmalek Aboussoror ${ }^{1}$ And Abdelatif Mansouri $^{2}$
}

\begin{abstract}
In this paper, which is an extension of [4], we first show the existence of solutions to a class of Min Sup problems with linked constraints, which satisfy a certain property. Then, we apply our result to a class of weak nonlinear bilevel problems. Furthermore, for such a class of bilevel problems, we give a relationship with appropriate d.c. problems concerning the existence of solutions.
\end{abstract}

Keywords. Min Sup problems, variational convergence, bilevel programming, d.c. programming.

Mathematics Subject Classification. 49K35, 90C47, 91A44, $46 \mathrm{~N} 10$.

\section{INTRODUCTION}

In this paper, we are firstly concerned by the following Min Sup problem with linked constraints (linked variables)

$$
\text { (S) } \operatorname{Min}_{x \in X} \sup _{y \in \mathcal{M}(x)} F(x, y)
$$

where

$$
F: X \times Y \rightarrow \mathbb{R} \quad \text { and } \quad \mathcal{M}: X \rightrightarrows Y
$$

Received September 1, 2006. Accepted November 11, 2007.

${ }^{1}$ Université Cadi Ayyade, Faculté Polydisciplinaire de Safi, B.P. 4162, Safi, Morocco;

aboussororabdel@hotmail.com

${ }^{2}$ Université Cadi Ayyad, Faculté des sciences Semlalia, Département de Mathématiques,

B.P. 2390, Marrakech, Morocco; mansouri@ucam.ac.ma 
are respectively a real valued function and a multifunction, $X$ is a compact subset of $\mathbb{R}^{p}$ and $Y$ is a compact convex subset of $\mathbb{R}^{q}$. For $x \in X$, set

$$
w(x)=\operatorname{Sup}_{y \in \mathcal{M}(x)} F(x, y) \quad \text { and } \quad v=\operatorname{Inf}_{x \in X} w(x) .
$$

Definition 1.1. A point $x$ in $X$ is said to be a min sup solution of $(S)$ if $w(x)=v$.

In the sequel, for simplicity, we will use the term of solution instead of min sup solution.

This paper is an extension of a recent work appeared in [4], which deals with Min Sup and weak bilevel problems. First of all, let us give some comments about some works dealing with Min Sup problems. Mention that the most studies relating to Min Sup problems have been devoted to the separate constraints case and finite Min Sup problems. Falk in [11], considered a linear max-min problem with linked constraints, for which under a basic assumption (the feasible region is bounded) he presented an algorithm. This algorithm is based on the branch and bound philosophy and gives the solution in a finite number of steps. Furthermore, he showed that there exists a solution which is a vertex point of a linear polyhedron. However, no computational experience has been given for large problems. Lignola and Morgan in [12], considered a more general formulation where the constraint $\mathcal{M}(x)$ is replaced by a parameterized constraint $\mathcal{M}(t, x)$ ( $t$ is the parameter). In particular, they have given sufficient conditions using the notion of $\Gamma$-limits [8], ensuring the existence of solutions to a class of problems having the form of $(S)$. Shimizu and Aiyoshi in [20], have given necessary conditions and computational methods for solutions under linked constraints via the case with separate constraints. The passage from the separate constraint case to the linked constraint case is deduced by the use of duality theory of convex programming. In this paper, we first consider a Min Sup problem $(S)$ with linked constraints. Under some sufficient conditions and a basic assumption $(\mathcal{P})$, we show that the problem $(S)$ admits solutions. As an application, we obtain the result given in [2] for a class of weak nonlinear bilevel problems. Indeed, this class corresponds to the case where $\mathcal{M}(x)$ is a set of solutions to another optimization problem parameterized by $x$. This illustrates of course the applicability of the first general study given for Min Sup problems. Note that such a class of nonlinear bilevel problems presents a major difficulty in finding sufficient conditions that ensure the existence of solutions (comments and an exhaustive list are given in [9]). Sufficient condition for the existence of solutions to weak bilevel problems are given in [1-3,16]. Finally, we give some cases where the property $(\mathcal{P})$ introduced for Min Sup problems is satisfied. As a consequence, the existence of solutions to the previous class of weak nonlinear bilevel problems can be deduced from the existence of solutions of appropriate d.c. problems. This, probably gives other possibilities in the invetigation of the existence of solutions for weak nonlinear bilevel optimization.

The remainder of the paper is organized as follows. In Section 2, we recall some definitions and give preliminary results. Then, we establish our main result on the existence of solutions to the Min Sup problem $(S)$. In Section 3, we apply 
our result to a class of weak nonlinear bilevel problems. In Section 4, we first present some cases where property $(\mathcal{P})$ is satisfied. As a consequence, we give a relationship between weak bilevel and d.c. problems. Finally, in Section 5, we give some concluding remarks and comments.

\section{PRELIMINARIES AND MAIN RESUlts}

In this section, we give sufficient conditions that ensure the existence of solutions to the problem $(S)$. Let

$$
\mathcal{M}_{n}: X \rightrightarrows Y, n \in \mathbb{N}
$$

be a sequence of multifunctions from $X$ to $Y$. In the sequel, the sets $X$ and $Y$ are respectively endowed with the induced topologies of $\mathbb{R}^{p}$ and $\mathbb{R}^{q}$.

Definition 2.1. Let $\left(A_{n}\right)_{n \in \mathbb{N}}$ be sequence of subsets of $Y$. Then, the sets $\operatorname{Lim} \sup A_{n}$ and $\operatorname{Liminf} A_{n}$ are respectively defined as $n \rightarrow+\infty$

$$
\begin{gathered}
\operatorname{Limsup}_{n \rightarrow+\infty} A_{n}=\left\{y \in Y / y=\lim _{k \rightarrow+\infty} y_{n_{k}}, y_{n_{k}} \in A_{n_{k}},\left(n_{k}\right)\right. \\
\text { is an infinite subsequence of integers }\} \\
\operatorname{Liminf}_{n \rightarrow+\infty} A_{n}=\left\{y \in Y / \exists y_{n} \in A_{n}, \forall n \in \mathbb{N}, \text { and } y=\lim _{k \rightarrow+\infty} y_{n}\right\} .
\end{gathered}
$$

In order to establish our results, we will need the following assumptions.

(2.1) For any $(n, x) \in \mathbb{N} \times X$, and any sequence $\left(x_{k}\right)$ converging to $x$ in $X$, we have

$$
\mathcal{M}_{n}(x) \subset \operatorname{Liminf}_{k \rightarrow+\infty} \mathcal{M}_{n}\left(x_{k}\right),
$$

that is the multifunction $\mathcal{M}_{n}($.$) is lower semicontinuous.$

(2.2) The function $F$ is continuous on $X \times Y$.

(2.3) For any $x \in X$, and any sequence $\left(x_{n}\right)$ converging to $x$ in $X$, we have

$$
\operatorname{Limsup}_{n \rightarrow+\infty} \mathcal{M}_{n}\left(x_{n}\right) \subset \mathcal{M}(x) .
$$

(2.4) For any $x \in X$, the function $F(x,$.$) is concave on Y$.

(2.5) For any $(n, x) \in \mathbb{N} \times X, \mathcal{M}(x) \subset \mathcal{M}_{n}(x)$.

(2.6) For any $n \in \mathbb{N}$, the multifunction $\mathcal{M}_{n}$ is closed valued.

For $n \in \mathbb{N}$, let us consider the following problem,

$$
\left(S_{n}\right) \quad \operatorname{Min}_{x \in X} \sup _{y \in \mathcal{M}_{n}(x)} F(x, y),
$$

and define on $X$, the function $w_{n}$ by

$$
w_{n}(x)=\operatorname{Sup}_{y \in \mathcal{M}_{n}(x)} F(x, y) .
$$

We begin by the following result on the existence of solutions to the problem $\left(S_{n}\right)$. 
Theorem 2.1. $\quad$ Let assumptions (2.1) and (2.2) be satisfied. Then, for any $n \in \mathbb{N}$, the problem $\left(S_{n}\right)$ has at least one solution.

Proof. See for example $[5,13]$.

Definition 2.2. Let assumptions (2.1) and (2.2) hold. For $n \in \mathbb{N}$, let $\bar{x}_{n}$ be a solution to the problem $\left(S_{n}\right)$. Let $\mathcal{A}$ denote the set of accumulation points of the sequence $\left(\bar{x}_{n}\right)$ (which is not necessarily reduced to a singleton). Let $\bar{x} \in \mathcal{A}$ and $\left(\bar{x}_{n_{k}}\right), k \in \mathbb{N}$, be a subsequence converging to $\bar{x}$, as $k \rightarrow+\infty$. Define the sequence

$$
\hat{x}_{n}=\bar{x}_{n_{k}} \quad \text { for } n_{k} \leq n<n_{k+1} .
$$

Then, we have $\hat{x}_{n} \rightarrow \bar{x}$, as $n \rightarrow+\infty$.

Now, let us define on $Y$ the following functions

$$
\phi_{\bar{x}}(y)=-F(\bar{x}, y)+\chi_{\mathcal{M}(\bar{x})}(y) \quad \text { and } \quad \phi_{n}(y)=-F\left(\hat{x}_{n}, y\right)+\chi_{\mathcal{M}_{n}\left(\hat{x}_{n}\right)}(y),
$$

where for a subset $Z$ of $Y, \chi_{Z}$ denotes the indicator function of $Z$, that is

$$
\begin{cases}\chi_{Z}(y)=0 & \text { if } y \in Z \\ \chi_{Z}(y)=+\infty & \text { if } y \notin Z .\end{cases}
$$

Let $\left(h_{n}\right)_{n \in \mathbb{N}}$ be a sequence of functions from $Y$ into $\overline{\mathbb{R}}=\mathbb{R} \cup\{-\infty,+\infty\}$. We recall the following definition (see $[6,10]$ ).

Definition 2.3. We say that the sequence $\left(h_{n}\right)_{n \in \mathbb{N}}$ variationally converges to $h_{0}$, if

(i) for any $y$ in $Y$, and any sequence $\left(y_{n}\right)_{n \in \mathbb{N}}$ converging to $y$ in $Y$, we have

$$
\liminf _{n \rightarrow+\infty} h_{n}\left(y_{n}\right) \geq h_{0}(y)
$$

(ii) for any $y$ in $Y$, there exists a sequence $\left(y_{n}\right)_{n \in \mathbb{N}}$ in $Y$, such that

$$
\limsup _{n \rightarrow+\infty} h_{n}\left(y_{n}\right) \leq h_{0}(y) .
$$

In the remainder of this section, and as quoted above in Definition $2.2, \bar{x} \in \mathcal{A}$ is an accumulation point of the fixed sequence $\left(\bar{x}_{n}\right)$, and $\left(\hat{x}_{n}\right)$ is the extended sequence of $\left(\bar{x}_{n}\right)$ which converges to $\bar{x}$.

Proposition 2.1. Let assumptions (2.1)-(2.3) hold. Then, for any $y \in Y$ and $\left(y_{n}\right)_{n \in \mathbb{N}}$ be a sequence converging to $y$ in $Y$, we have

$$
\liminf _{n \rightarrow+\infty} \phi_{n}\left(y_{n}\right) \geq \phi_{\bar{x}}(y)
$$

Proof. Let $y \in Y$ and $\left(y_{n}\right)_{n \in \mathbf{N}}$ a sequence converging to $y$ in $Y$. We need to consider the following two cases.

(1) If $y \in \mathcal{M}(\bar{x})$, then $\phi_{\bar{x}}(y)=-F(\bar{x}, y)$, and

$$
\liminf _{n \rightarrow+\infty} \phi_{n}\left(y_{n}\right) \geq \lim _{n \rightarrow+\infty}\left\{-F\left(\hat{x}_{n}, y_{n}\right)\right\}=-F(\bar{x}, y)=\phi_{\bar{x}}(y) .
$$


(2) If $y \notin \mathcal{M}(\bar{x})$, then $\phi_{\bar{x}}(y)=+\infty$. Furthermore, we have $y_{n} \notin \mathcal{M}_{n}\left(\hat{x}_{n}\right)$, for large $n \in \mathbb{N}$. Otherwise, there exists an infinite subset $\mathcal{N}_{1}$ of $\mathbb{N}$, such that $y_{n} \in \mathcal{M}_{n}\left(\hat{x}_{n}\right)$, for all $n \in \mathcal{N}_{1}$. Then, by using assumption (2.3), we get

$$
y \in \operatorname{Limsup}_{n \rightarrow+\infty} \mathcal{M}_{n}\left(\hat{x}_{n}\right) \subset \mathcal{M}(\bar{x}),
$$

and the contradiction. Hence, $y_{n} \notin \mathcal{M}_{n}\left(\hat{x}_{n}\right)$, for large $n \in \mathbb{N}$. It follows that $\phi_{n}\left(y_{n}\right)=+\infty$, for large $n \in \mathbb{N}$, and the result is obvious.

For $x \in X$, set $F_{x}()=.F(x,$.$) , and consider the following property which is our$ basic assumption in the remainder of this section

$$
\mathcal{I}_{x}=\left\{y \in Y / F_{x}^{\prime}(y ; z-y) \leq 0, \forall z \in Y\right\} \cap \mathcal{M}(x) \neq \emptyset, \quad \forall x \in X
$$

where $F_{x}^{\prime}(y ; d)$ denotes the directional derivative of the function $F_{x}($.$) at y$ in the direction $d \in \mathbb{R}^{q} \backslash\{0\}$, that is

$$
F_{x}^{\prime}(y ; d)=\lim _{t \searrow 0^{+}} \frac{F_{x}(y+t d)-F_{x}(y)}{t} .
$$

Remark 2.1. The property $(\mathcal{P})$ means that for any $x \in X$, the solution set of the problem

$$
S(x) \quad \operatorname{Max}_{y \in Y} F(x, y)
$$

and the constraint set $\mathcal{M}(x)$ have a nonempty intersection.

Proposition 2.2. Let assumptions (2.1)-(2.5) hold. If moreover, the property $(\mathcal{P})$ is satisfied, then for any $y \in Y$, there exists a sequence $\left(y_{n}\right)_{n \in \mathbb{N}}$ in $Y$, such that

$$
\limsup _{n \rightarrow+\infty} \phi_{n}\left(y_{n}\right) \leq \phi_{\bar{x}}(y)
$$

Proof. Let $y \in Y$. Then, as above, we distinguish the following two cases.

(1) If $y \in \mathcal{M}(\bar{x})$, then according to property $(\mathcal{P})$, for $n \in \mathbb{N}$, there exists $y_{n} \in \mathcal{I}_{\hat{x}_{n}}$. So, $y_{n} \in \mathcal{M}\left(\hat{x}_{n}\right)$, and since $F_{\hat{x}_{n}}^{\prime}\left(y_{n}, z-y_{n}\right) \leq 0$, for all $z \in Y$, it follows that $y_{n}$ is a solution to the problem

$$
\operatorname{Max}_{y \in Y} F_{\hat{x}_{n}}(y)
$$

Furthermore, by using assumption (2.5), we have $y_{n} \in \mathcal{M}_{n}\left(\hat{x}_{n}\right)$, for all $n \in \mathbb{N}$. Then,

$$
\limsup _{n \rightarrow+\infty} \phi_{n}\left(y_{n}\right)=\limsup _{n \rightarrow+\infty}\left\{-F\left(\hat{x}_{n}, y_{n}\right)\right\}=\lim _{\substack{n \rightarrow+\infty \\ n \in \mathcal{N}_{2}}}\left\{-F\left(\hat{x}_{n}, y_{n}\right)\right\}
$$

where $\mathcal{N}_{2}$ is an infinite subset of $\mathbb{N}$. Let $\mathcal{N}_{3} \subset \mathcal{N}_{2}$, such that $y_{n} \rightarrow \hat{y}$, as $n \rightarrow+\infty$, $n \in \mathcal{N}_{3}$. Assumption (2.3) implies that $\hat{y} \in \mathcal{M}(\bar{x})$. Let us show that $\hat{y}$ is a solution 
of the problem $\operatorname{Max}_{y \in \mathcal{M}(\bar{x})} F_{\bar{x}}(y)$. First, let us show that $\hat{y}$ is a solution of the problem $\operatorname{Max}_{y \in Y} F_{\bar{x}}(y)$. Suppose that there exists $y^{0} \in Y$, such that

$$
F(\bar{x}, \hat{y})<F\left(\bar{x}, y^{0}\right)
$$

Hence

$$
F\left(\hat{x}_{n}, y_{n}\right)<F\left(\hat{x}_{n}, y^{0}\right)
$$

for large $n \in \mathcal{N}_{3}$. This, contradicts the the optimality of $y_{n}$ to the problem

$$
\operatorname{Max}_{y \in Y} F_{\hat{x}_{n}}(y), \quad n \in \mathcal{N}_{3} .
$$

Therefore, $\hat{y}$ is a solution of the problem $\operatorname{Max}_{y \in Y} F_{\bar{x}}(y)$, and since $\hat{y} \in \mathcal{M}(\bar{x})$, it follows that $\hat{y}$ is a solution of the problem $\operatorname{Max}_{y \in \mathcal{M}(\bar{x})} F_{\bar{x}}(y)$. Finally, we get

$$
\lim _{\substack{n \rightarrow+\infty \\ n \in \mathcal{N}_{2}}}\left\{-F\left(\hat{x}_{n}, y_{n}\right)\right\}=\lim _{\substack{n \rightarrow+\infty \\ n \in \mathcal{N}_{3}}}\left\{-F\left(\hat{x}_{n}, y_{n}\right)\right\}=-F(\bar{x}, \hat{y}) \leq-F(\bar{x}, y)=\phi_{\bar{x}}(y) .
$$

That is

$$
\limsup _{n \rightarrow+\infty} \phi_{n}\left(y_{n}\right) \leq \phi_{\bar{x}}(y) .
$$

(2) If $y \notin \mathcal{M}(\bar{x})$, then $\phi_{\bar{x}}(y)=+\infty$, and the result is obvious.

The following convergence result is an immediate consequence of Propositions 2.1 and 2.2.

Corollary 2.1. Let assumptions (2.1)-(2.5) hold. If moreover, the property $(\mathcal{P})$ is satisfied, then the sequence $\left(\phi_{n}\right)_{n \in \mathbb{N}}$, variationally converges to $\phi_{\bar{x}}$.

For $n \in \mathbb{N}$, and $x \in X$, consider the problems

$$
\mathcal{Q}_{n}(x) \quad \operatorname{Max}_{y \in \mathcal{M}_{n}(x)} F_{x}(y) \text { and } \mathcal{Q}(x) \operatorname{Max}_{y \in \mathcal{M}(x)} F_{x}(y)
$$

and denote by $\operatorname{Argmax} \mathcal{Q}_{n}(x)$ and $\operatorname{Argmax} \mathcal{Q}(x)$, the sets of solutions to the problems $\mathcal{Q}_{n}(x)$ and $\mathcal{Q}(x)$ respectively. Then, we have the following important convergence result.

Corollary 2.2. Let assumptions (2.1)-(2.5) hold. If the property $(\mathcal{P})$ is satisfied, then

$$
\underset{n \rightarrow+\infty}{\operatorname{Limsup}} \operatorname{Argmax} \mathcal{Q}_{n}\left(\hat{x}_{n}\right) \subset \operatorname{Argmax} \mathcal{Q}(\bar{x})
$$

Proof. Since the sequence $\left(\phi_{n}\right)_{n \in \mathbb{N}}$ variationally converges to $\phi_{\bar{x}}$, then $($ see $[6,10])$

$$
\underset{n \rightarrow+\infty}{\operatorname{Limsup}} \operatorname{Argmax} \mathcal{Q}_{n}\left(\hat{x}_{n}\right) \subset \operatorname{Argmax} \mathcal{Q}(\bar{x}) .
$$

Set $\left\{\bar{x}_{n_{k}}, k \in \mathbb{N}\right\}=\left\{\hat{x}_{n}, n \in \mathcal{N}_{0}^{*}\right\}$, where $\mathcal{N}_{0}^{*}$ be an infinite subset of $\mathbb{N}$, such that $\hat{x}_{n}=\bar{x}_{n}$, for all $n \in \mathcal{N}_{0}^{*}$.

Now, we are in a position to give our main result in this section concerning the existence of solution to the problem $(S)$. 
Theorem 2.2. Let assumptions (2.1)-(2.6) hold. If the property $(\mathcal{P})$ is satisfied, then the problem $(S)$ has at least one solution.

Proof. We will show that the accumulation point $\bar{x}$ (which has been chosen arbitrary) is a solution to $(S)$. Suppose that there exists $x_{0} \in X$, such that

$$
w\left(x_{0}\right)<w(\bar{x})
$$

(recall that for $\left.x \in X, w(x)=\operatorname{Sup}_{y \in \mathcal{M}(x)} F(x, y)\right)$. Then,

$$
F\left(x_{0}, y\right)<w(\bar{x}), \quad \forall y \in \mathcal{M}\left(x_{0}\right) .
$$

For $n \in \mathcal{N}_{0}^{*}\left(\mathcal{N}_{0}^{*}\right.$ is the set introduced above), let $\bar{y}_{n}$ be a solution to the problem

$$
\operatorname{Max}_{y \in \mathcal{M}_{n}\left(\bar{x}_{n}\right)} F\left(\bar{x}_{n}, y\right)
$$

(such a point exists by using assumption (2.6), the compactness of $Y$ and the continuity of the function $\left.F\left(\bar{x}_{n},.\right)\right)$. That is $w_{n}\left(\bar{x}_{n}\right)=F\left(\bar{x}_{n}, \bar{y}_{n}\right)$, and $\bar{y}_{n} \in$ $\operatorname{Argmax} \mathcal{Q}_{n}\left(\bar{x}_{n}\right)$. Since $Y$ is compact, there exists an infinite subset $\mathcal{N}_{1}^{*}$ of $\mathcal{N}_{0}^{*}$, such that $\bar{y}_{n} \rightarrow \bar{y}, n \in \mathcal{N}_{1}^{*}$. Hence, by Corollary 2.2, we have $\bar{y} \in \operatorname{Argmax} \mathcal{Q}(\bar{x})$. That is $w(\bar{x})=F(\bar{x}, \bar{y})$. For $n \in \mathcal{N}_{1}^{*}$, let $y_{n}^{0}$ be a solution to the problem

$$
\operatorname{Max}_{y \in \mathcal{M}_{n}\left(x_{0}\right)} F\left(x_{0}, y\right)
$$

That is $w_{n}\left(x_{0}\right)=F\left(x_{0}, y_{n}^{0}\right)$. By the same arguments as in the above, there exists an infinite subset $\mathcal{N}_{2}^{*}$ of $\mathcal{N}_{1}^{*}$, such that $y_{n}^{0} \rightarrow y_{0}, n \in \mathcal{N}_{2}^{*}$, and $y_{0} \in \mathcal{M}\left(x_{0}\right)$ (by assumption (2.3)). Then,

$$
F\left(x_{0}, y_{0}\right)<w(\bar{x})=F(\bar{x}, \bar{y}) .
$$

Hence

$$
F\left(x_{0}, y_{n}^{0}\right)<F\left(\bar{x}_{n}, \bar{y}_{n}\right), \quad \text { for large } n, n \in \mathcal{N}_{2}^{*} .
$$

It follows that

$$
w_{n}\left(x_{0}\right)<w_{n}\left(\bar{x}_{n}\right), \quad \text { for large } n, n \in \mathcal{N}_{2}^{*} .
$$

This contradicts the optimality of $\bar{x}_{n}$ to the problem $\left(S_{n}\right)$, for large $n, n \in \mathcal{N}_{2}^{*}$, and the proof is complete.

Let us consider the following simple example where assumptions (2.1)-(2.6) and the property $(\mathcal{P})$ are satisfied.

Example 2.1. Let $X=[1,2] \subset \mathbb{R}, Y=[1,3] \subset \mathbb{R}$, and $F(x, y)=-(x-1) y^{2}$. Let $\mathcal{M}$ and $\mathcal{M}_{n}, n \in \mathbb{N}$, be the multifunctions defined by

$$
\mathcal{M}(x)=[1, x] \text { and } \mathcal{M}_{n}(x)=[1, x+1 /(n+1)] .
$$


We easily verify that assumptions (2.1)-(2.6) are satisfied. Let us verify the property $(\mathcal{P})$. We have

$$
\begin{gathered}
F_{x}^{\prime}(y ; z-y)=\frac{\partial F}{\partial y}(x, y) \times(z-y)=-2(x-1)(z-y) y \\
\left\{y \in Y / F_{x}^{\prime}(y ; z-y) \leq 0, \forall z \in Y\right\}= \begin{cases}{[1,3]} & \text { if } x=1 \\
\{1\} & \text { if } x \in] 1,2] .\end{cases} \\
\text { Then, we have } \mathcal{I}_{x}= \begin{cases}{[1,3] \cap \mathcal{M}(1)=\{1\}} & \text { if } x=1 \\
\{1\} \cap \mathcal{M}(x)=\{1\} & \text { if } x \in] 1,2] .\end{cases}
\end{gathered}
$$

Thus, property $(\mathcal{P})$ is satisfied.

In the following example, we will see a problem which admits solutions and belongs to the class of Min Sup problems satisfying assumptions (2.1) to (2.6), but that does not satisfy the property $(\mathcal{P})$. Therefore, property $(\mathcal{P})$ is not a necessary condition for the existence of solutions.

Example 2.2. Let $X=Y=[1,2] \subset \mathbb{R}, F(x, y)=x\left(3-y^{2}\right)+x$, and for $x \in X$, let $\mathcal{M}(x)$ denote the set of solutions to the parameterized problem

$$
\mathcal{P}(x) \quad \operatorname{Min}_{y \in Y}(1-x) y .
$$

For $\epsilon_{n} \searrow 0^{+}$, let $\mathcal{M}_{n}(x)$ denote the set of $\epsilon_{n}$-approximate solutions to the problem $\mathcal{P}(x)$. Then, it is easy to verify that assumptions (2.2), (2.4), (2.5) and (2.6) are satisfied. Furthermore, using the results given in [15], we get the following properties:

(i) for any $(n, x) \in \mathbb{N} \times X$, and any sequence $\left(x_{k}\right)$ converging to $x$ in $X$, we have

$$
\mathcal{M}_{n}(x) \subset \operatorname{Liminf}_{k \rightarrow+\infty} \mathcal{M}_{n}\left(x_{k}\right) ;
$$

(ii) for any $x \in X$, and any sequence $\left(x_{n}\right)$ converging to $x$ in $X$, we have

$$
\operatorname{Limsup}_{n \rightarrow+\infty} \mathcal{M}_{n}\left(x_{n}\right) \subset \mathcal{M}(x)
$$

that is assumptions (2.1) and (2.3) are satisfied. On the other hand, we have

$$
\mathcal{M}(x)=\left\{\begin{array}{ll}
{[1,2]} & \text { if } x=1 \\
\{2\} & \text { if } x \in] 1,2]
\end{array} \quad \text { and } \quad w(x)=\sup _{y \in \mathcal{M}(x)} F(x, y)= \begin{cases}3 & \text { if } x=1 \\
0 & \text { if } x \in] 1,2] .\end{cases}\right.
$$

Then, $v=\inf _{x \in X} w(x)=0$, and hence any $\left.\left.x \in\right] 1,2\right]$ is a solution of $(S)$. Therefore, the problem $(S)$ satisfies assumptions (2.1) to (2.6), and admits solutions. However, we have

$$
\left\{y \in Y / F_{x}^{\prime}(y ; z-y) \leq 0, \forall z \in Y\right\}=\{1\}, \text { for all } x \in[1,2] .
$$

Then, we have $\mathcal{I}_{x}=\emptyset$, for $\left.\left.x \in\right] 1,2\right]$. That is property $(\mathcal{P})$ is not satisfied. 
Remark 2.2. Remark that even though assumptions (2.1), (2.3) and (2.5) are satisfied in Example 2.2., the multifunction $\mathcal{M}($.$) is not lower semicontinuous at$ $x=1$. Such a property is known as a very restrective assumption and is rarely satisfied by the solution set.

Example 2.3. It is important to note that a large class of problems, the so-called linear max-min problems (or min-max) with a bounded feasible region, always satisfy assumptions (2.1) to (2.6). In fact, let the following max-min problem $($ see $[11])$

$$
\operatorname{Max}_{x \in\left(\mathbb{R}_{+}\right)^{p}} \min _{\substack{y \in\left(\mathbf{R}_{+}\right)^{q} \\ A x+B y \leq b}}[\langle c, x\rangle+\langle d, y\rangle]
$$

where $c \in \mathbb{R}^{p}, d \in \mathbb{R}^{q}, b \in \mathbb{R}^{r}, A \in \mathbb{R}^{r \times p}$, and $B \in \mathbb{R}^{r \times q}$. Set

$$
\begin{gathered}
\mathcal{X}=\left\{x \in\left(\mathbb{R}_{+}\right)^{p} / \exists y \geq 0, A x+B y \leq b\right\} \text { and } \\
\mathcal{Y}=\left\{y \in\left(\mathbb{R}_{+}\right)^{q} / \exists x \geq 0, A x+B y \leq b\right\}
\end{gathered}
$$

which are the projections of the feasible region $\mathcal{R}=\left\{(x, y) \in\left(\mathbb{R}_{+}\right)^{p} \times\left(\mathbb{R}_{+}\right)^{q} / A x+\right.$ $B y \leq b\}$ onto $\mathbb{R}^{p}$ and $\mathbb{R}^{q}$ respectively. Falk in [11], assumes that $\mathcal{R}$ is a compact set. Hence, both $\mathcal{X}$ and $\mathcal{Y}$ are compact (as projections of compact sets) and also convex. On the other hand, the problem $(S)$ can be written as (see Th. $2.1[11]$ )

$$
\operatorname{Max}_{x \in \mathcal{X}} \min _{y \in \mathcal{M}(x)}[\langle c, x\rangle+\langle d, y\rangle]
$$

where

$$
\mathcal{M}(x)=\left\{y \in\left(\mathbb{R}_{+}\right)^{q} / B y \leq b-A x\right\}
$$

which is a subset of $\mathcal{Y}$. Define $g(x, y)=A x+B y-b, g_{i}(x, y)=A_{i} x+B_{i} y-b_{i}$, where $A_{i}, B_{i}$ denote the $i^{\text {th }}$ rows of $A, B$ respectively, and $b_{i}$ the $i^{\text {th }}$ component of $b$. For $i \in\{1, \ldots, r\}$ and $n \in \mathbb{N}$, set

$$
\mathcal{M}_{i}(x)=\left\{y \in\left(\mathbb{R}_{+}\right)^{q} / g_{i}(x, y) \leq 0\right\}, \mathcal{M}_{i, n}(x)=\left\{y \in\left(\mathbb{R}_{+}\right)^{q} / g_{i}(x, y) \leq \frac{1}{n+1}\right\}
$$

Then, we have $\mathcal{M}(x)=\bigcap_{i=1, \ldots, r} \mathcal{M}_{i}(x)$. Set $\mathcal{M}_{n}(x)=\bigcap_{i=1, \ldots, r} \mathcal{M}_{i, n}(x)$. It is easy to see that assumptions (2.2), (2.4), (2.5) and (2.6) are satisfied. For the verification of assumption (2.1), see for example, Corollary 3.2.3.2 [7]. Let us verify assumption (2.3). Let $x \in \mathcal{X}$, and $x_{n} \rightarrow x$ in $\mathcal{X}$, as $n \rightarrow+\infty$. Let $y \in$ $\operatorname{Lim} \sup _{n \rightarrow+\infty} \mathcal{M}_{n}\left(x_{n}\right)$. There exists $y_{n} \rightarrow y$ in $\mathcal{Y}$, as $n \rightarrow+\infty$, and $y_{n} \in \mathcal{M}_{n}\left(x_{n}\right)$, $\forall n \in \mathcal{N}$, where $\mathcal{N}$ is an infinite subset of $\mathbb{N}$. That is $g_{i}\left(x_{n}, y_{n}\right) \leq \frac{1}{n+1}$, for all $i$. Then, for all $i$, by the continuity of $g_{i}$, we obtain $g_{i}(x, y) \leq 0$. That is $y \in \mathcal{M}(x)$. Furthermore, Falk showed that the problem $(S)$ admits solutions in the sense of Definition 1.1. However, there is no conditions which guarantee property $(\mathcal{P})$. 


\section{Application to Weak nONLINEAR Bilevel PROBlems}

In this section, in order to illustrate the applicability of the previous study given in Section 2, we consider a class of weak nonlinear bilevel problems for which sufficient conditions ensuring the existence of solutions have been given in [2]. Indeed, this result will be deduced by application of our Theorem 2.2 given in Section 2 for Min Sup problems, in the case where $\mathcal{M}(x)$ is a set of solutions to another optimization problem parameterized by $x$.

Let us consider a static uncooperative two-player game in which one player has the leadership (called the leader) and the second one reacts optimally (called the follower). The leader with an objective function $\tilde{F}$ and a set of strategies $\tilde{X} \subset \mathbb{R}^{p}$, knows the objective function $\tilde{f}$ and the set of strategies $\tilde{Y} \subset \mathbb{R}^{q}$, of the follower, but he can not influence his choice. In the pessimistic case, the leader has to consider the following optimization problem

$$
(\tilde{S}) \quad \operatorname{Min}_{x \in \tilde{X}} \sup _{y \in \tilde{\mathcal{M}}(x)} \tilde{F}(x, y)
$$

where $\tilde{\mathcal{M}}(x)$ is the set of solutions to the lower level problem

$$
\tilde{P}(x) \quad \operatorname{Min}_{y \in \tilde{Y}} \tilde{f}(x, y)
$$

and

$$
\tilde{F}, \tilde{f}: \tilde{X} \times \tilde{Y} \rightarrow \mathbb{R} .
$$

As mentioned in the introduction, weak nonlinear bilevel problems present a major difficulty in finding mild sufficient conditions that ensure the existence of solutions. In general, such problems present difficulties in their survey in both numerical and theoretical framework, and therefore they are less considered in the literature.

In the sequel, we assume that $\tilde{X}$ and $\tilde{Y}$ are a compact and a compact convex subsets of $\mathbb{R}^{p}$ and $\mathbb{R}^{q}$ respectively, and are respectively equipped with the induced topologies of $\mathbb{R}^{p}$ and $\mathbb{R}^{q}$.

For $\epsilon>0$, we consider the following regularized problem of $(\tilde{S})$ (see [15])

$$
\left(\tilde{S}_{\epsilon}\right) \quad \operatorname{Min}_{x \in \tilde{X}} \sup _{y \in \tilde{\mathcal{M}}_{\epsilon}(x)} \tilde{F}(x, y)
$$

where $\tilde{\mathcal{M}}_{\epsilon}(x)$ is the set of $\epsilon$-approximate solutions of $\tilde{P}(x)$. The following assumptions will be used $([2])$

(3.1) For any $(x, y) \in \tilde{X} \times \tilde{Y}$, and any sequence $\left(x_{n}\right)$ converging to $x$ in $\tilde{X}$, there exists a sequence $\left(y_{n}\right)$ converging to $y$ in $\tilde{Y}$, such that

$$
\limsup _{n \rightarrow+\infty} \tilde{f}\left(x_{n}, y_{n}\right) \leq \tilde{f}(x, y)
$$


(3.2) For any $x \in \tilde{X}$, the function $\tilde{f}(x,$.$) is convex on \tilde{Y}$.

(3.3) The function $\tilde{f}$ is lower semi-continuous on $\tilde{X} \times \tilde{Y}$.

(3.4) The function $\tilde{F}$ is continuous on $\tilde{X} \times \tilde{Y}$.

For $\epsilon_{n} \searrow 0^{+}$, set $\tilde{\mathcal{M}}_{\epsilon_{n}}(x)=\tilde{\mathcal{M}}_{n}(x)$ and denote $\left(\tilde{S}_{\epsilon_{n}}\right)$ by $\left(\tilde{S}_{n}\right)$.

Proposition 3.1. Let assumptions (3.1)-(3.3) hold. Then,

(1) for any $(n, x) \in \mathbb{N} \times \tilde{X}$, and any sequence $\left(x_{k}\right)$ converging to $x$ in $\tilde{X}$, we have

$$
\tilde{\mathcal{M}}_{n}(x) \subset \operatorname{Liminf}_{k \rightarrow+\infty} \tilde{\mathcal{M}}_{n}\left(x_{k}\right)
$$

(2) for any $x \in \tilde{X}$, and any sequence $\left(x_{n}\right)$ converging to $x$ in $\tilde{X}$, we have

(i) $\operatorname{Lim} \sup _{n \rightarrow+\infty} \tilde{\mathcal{M}}_{n}\left(x_{n}\right) \subset \tilde{\mathcal{M}}(x)$,

(ii) $\operatorname{Lim} \sup _{n \rightarrow+\infty} \tilde{\mathcal{M}}_{\epsilon}\left(x_{n}\right) \subset \tilde{\mathcal{M}}_{\epsilon}(x), \forall \epsilon \geq 0$.

Proof. For 1) and i) of 2) see [15]. The proof of (ii) is easily deduced from the proof of (i).

Theorem 3.1. Let assumptions (3.1)-(3.4) hold. Then, for any $n \in \mathbb{N}$, the problem $\left(\tilde{S}_{n}\right)$ has at least one solution.

Proof. Apply Theorem 2.1 or see [15].

For $x \in \tilde{X}$, set $\tilde{F}_{x}()=.\tilde{F}(x,$.$) and \tilde{f}_{x}()=.\tilde{f}(x,$.$) . Then, we have the following$ result on the existence of solutions to the problem $(\tilde{S})$.

Theorem 3.2. Let assumptions (3.1)-(3.4) hold. If moreover, the following assumptions are satisfied,

(3.5) For any $x \in \tilde{X}$, the function $\tilde{F}(x,$.$) is concave on \tilde{Y}$,

(3.6) For any $x \in \tilde{X}, \tilde{\mathcal{I}}_{x}=\left\{y \in \tilde{Y} / \tilde{F}_{x}^{\prime}(y ; z-y) \leq 0, \forall z \in \tilde{Y}\right\} \cap\left\{y \in \tilde{Y} / \tilde{f}_{x}^{\prime}(y ; z-\right.$ y) $\geq 0, \forall z \in \tilde{Y}\} \neq \emptyset$,

then the problem $(\tilde{S})$ has at least one solution.

Proof. Apply Theorem 2.2 by remarking that assumption (3.6) corresponds to the property $(\mathcal{P})$.

Let the following example where all assumptions (3.1) to (3.6) are satisfied.

Example $3.1[16]$. Let $\tilde{X}=\tilde{Y}=[0,1], \tilde{F}(x, y)=\frac{1}{2}(1-x)+x y, \tilde{f}(x, y)=$ $(x-1) y$. We see that all assumptions (3.1) to (3.5) are satisfied. Furthermore, we have

$$
\tilde{\mathcal{M}}(x)= \begin{cases}{[0,1]} & \text { if } x=1 \\ \{1\} & \text { if } x \in[0,1[\end{cases}
$$

and

$$
\left\{y \in \tilde{Y} / \tilde{F}_{x}^{\prime}(y ; z-y) \leq 0, \forall z \in \tilde{Y}\right\}=\{1\}, \forall x \in[0,1]
$$

Hence for any $x \in \tilde{X}, \tilde{\mathcal{I}}_{x} \neq \emptyset$. Thus, assumption (3.6) is satisfied. 
Remark 3.1. Set $h_{x}=-\tilde{F}_{x}$. Then, assumption (3.6) means that the convex problems

$$
\operatorname{Min}_{y \in \tilde{Y}} \tilde{f}_{x}(y) \quad \text { and } \quad \operatorname{Min}_{y \in \tilde{Y}} h_{x}(y)
$$

have a common solution $\tilde{y}$, which in terms of subdifferential and normal cone, can be written as

$$
\forall x \in \tilde{X}, 0 \in\left(\partial h_{x}(\tilde{y})+\mathcal{N}_{\tilde{Y}}(\tilde{y}) \cap \partial \tilde{f}_{x}(\tilde{y})+\mathcal{N}_{\tilde{Y}}(\tilde{y})\right)
$$

where $\partial h_{x}(\tilde{y})$ and $\mathcal{N}_{\tilde{Y}}(\tilde{y})$ denote respectively the subdifferential of $h_{x}$ at $\tilde{y}$ and the normal cone to $\tilde{Y}$ at $\tilde{y}$, that is

$$
\begin{gathered}
\partial h_{x}(\tilde{y})=\left\{y^{*} \in \mathbb{R}^{q} / h_{x}(y) \geq h_{x}(\tilde{y})+\left\langle y^{*}, y-\tilde{y}\right\rangle, \forall y \in \mathbb{R}^{q}\right\} \text { and } \\
\mathcal{N}_{\tilde{Y}}(\tilde{y})=\left\{y^{*} \in \mathbb{R}^{q} /\left\langle y^{*}, z-\tilde{y}\right\rangle \leq 0, \forall z \in \tilde{Y}\right\} .
\end{gathered}
$$

Remark 3.2.

(1) Note that the weak linear bilevel problem considered in [3] which admits solutions under sufficient conditions satisfies assumptions (3.1) to (3.5).

(2) A simple case where assumptions (3.1) to (3.5) and the basic assumption (3.6) are satisfied (easy to verify) is that when the objective functions $\tilde{F}$ and $\tilde{f}$ are in the form

$$
\tilde{F}(x, y)=h(x)-k(y) \quad \tilde{f}(x, y)=g(x)+k(y)
$$

where $k: \mathbb{R}^{q} \rightarrow \mathbb{R}$, is a convex function, and $h, g: \mathbb{R}^{p} \rightarrow \mathbb{R}$, are continuous functions.

(3) It is well known that weak bilevel problems admit solutions under the lower semi-continuity of the follower's optimal solution set. In fact, it is not difficult to see that the marginal function $\tilde{w}(x)=\operatorname{Sup}_{y \in \tilde{\mathcal{M}}(x)} \tilde{F}(x, y)$, is lower semi-continuous on $\tilde{X}$, when $\tilde{F}$ and $\tilde{\mathcal{M}}($.$) are lower semi-continuous on \tilde{X} \times \tilde{Y}$ and $\tilde{X}$ respectively, and hence when $\tilde{X}$ is compact, we get the existence of solutions. However, such property is strong and difficult to obtain under mild assumptions. So, by the use of the results given in Section 2, the property $(\mathcal{P})$ gives another alternative for the existence of solutions, and obviously any relation between $(\mathcal{P})$ and lower semi-continuity of $\tilde{\mathcal{M}}($.$) , if it exists, must use some properties verified by \tilde{F}$, $\tilde{f}$ and the follower's constraint set simultaneously. But in general, there is no trivial implications between them (except perhaps under additional assumptions), as we can see in the following two examples.

Example 3.2. Let $\tilde{X}=[2,3], \tilde{Y}=[-5,5]$, and $\tilde{f}: \tilde{X} \times \tilde{Y} \rightarrow \mathbb{R}$, defined by

$$
f(x, y)= \begin{cases}0 & \text { if } y^{2}-x^{2} \leq 0 \\ y^{2}-x^{2} & \text { if } y^{2}-x^{2}>0\end{cases}
$$

Then, $\tilde{\mathcal{M}}(x)=[-x, x]=\{y \in[-5,5] /-x \leq y \leq x\}$, and hence $\tilde{\mathcal{M}}($.$) is lower$ semi-continuous on $\tilde{X}$. Let $\tilde{F}: \tilde{X} \times \tilde{Y} \rightarrow \mathbb{R}$, defined by $\tilde{F}(x, y)=(x-3)^{2} y$. Then, 
$F_{x}^{\prime}(y ; z-y)=(x-3)^{2}(z-y)$, and

$$
\left\{y \in \tilde{Y} / \tilde{F}_{x}^{\prime}(y ; z-y) \leq 0, \forall z \in \tilde{Y}\right\}= \begin{cases}{[-5,5]} & \text { if } x=3 \\ \{5\} & \text { if } x \in[2,3[.\end{cases}
$$

It Follows that for any $x \in\left[2,3\left[, \tilde{\mathcal{I}}_{x}=\{5\} \cap[-x, x]=\emptyset\right.\right.$. That is property $(\mathcal{P})$ is not satisfied.

Example 3.3. Let $\tilde{X}=\tilde{Y}=[1,2]$. Let $\tilde{\mathcal{M}}(x)$ denote the solution set of the lower level problem $\mathcal{P}(x)$ considered in Example 2.2. We have

$$
\tilde{\mathcal{M}}(x)= \begin{cases}{[1,2]} & \text { if } x=1 \\ \{2\} & \text { if } x \in] 1,2] .\end{cases}
$$

We easily verify that $\tilde{\mathcal{M}}($.$) is not lower semi-continuous at x=1$. Let $\tilde{F}: \tilde{X} \times$ $\tilde{Y} \rightarrow \mathbb{R}$, be the leader's objective function defined by $\tilde{F}(x, y)=(x-1) y$. Then, $F_{x}^{\prime}(y ; z-y)=(x-1)(z-y)$, and

$$
\left\{y \in \tilde{Y} / \tilde{F}_{x}^{\prime}(y ; z-y) \leq 0, \forall z \in \tilde{Y}\right\}= \begin{cases}{[1,2]} & \text { if } x=1 \\ \{2\} & \text { if } x \in] 1,2] .\end{cases}
$$

Then

$$
\tilde{\mathcal{I}}_{x}= \begin{cases}{[1,2]} & \text { if } x=1 \\ \{2\} & \text { if } x \in] 1,2]\end{cases}
$$

Hence property $(\mathcal{P})$ is satisfied.

Now, let us go back to Section 2. When $\mathcal{M}(x)$ represents a set of solutions to another optimization problem parameterized by $x$, as a consequence of the satisfaction of the property $(\mathcal{P})$, we obtain a relationship between weak bilevel and d.c. problems, as we will see in the following section.

\section{Existence of SOlutions to WeAK BileVEl PROBlems VIA \\ D.C. PROBLEMS}

In this section, we will present some cases where the property $(\mathcal{P})$ is satisfied. This allows us to deduce the existence of solutions to weak nonlinear bilevel problems from the existence of solutions to appropriate d.c. problems.

\subsection{Sufficient Conditions For SATisfying $(\mathcal{P})$}

With the notations and the data of Section 2, let $f: X \times Y \rightarrow \mathbb{R}$, be a function, and for $x \in X$, let $\mathcal{M}(x)$ denote the set of solutions to the following parameterized problem

$$
\mathcal{P}(x) \quad \operatorname{Min}_{y \in Y} f(x, y) .
$$

For $x \in X$, set $f(x,)=.f_{x}(),. F(x,)=.F_{x}($.$) and \hat{F}=-F$. In the remainder of this section, we assume that for any $x \in X, f_{x}($.$) and F_{x}($.$) are respectively a$ convex function and a concave function on the compact convex set $Y$. 
Then, we have the following proposition concerning the satisfaction of the prop$\operatorname{erty}(\mathcal{P})$.

Proposition 4.1. Assume that the following assumption is satisfied

(4.1) for any $x \in X$, there exists $\bar{y} \in Y$, such that

$$
0 \in \partial f_{x}(\bar{y}) \cap \partial \hat{F}_{x}(\bar{y}) .
$$

Then, the property $(\mathcal{P})$ is satisfied.

Proof. The proof is obvious, so it is omitted.

Remark 4.1. If for any $x \in X$, the functions $f_{x}($.$) and F_{x}($.$) are differentiable$ on $\mathbb{R}^{q}$, then assumption (4.1) becomes:

(4.1) $^{*}$ for any $x \in X$, there exists $\bar{y} \in Y$, such that $\nabla f_{x}(\bar{y})=\nabla \hat{F}_{x}(\bar{y})=0$

that is $\bar{y}=\left(\bar{y}_{1}, \ldots, \bar{y}_{q}\right)$ is a solution of a system of $2 q$ equations.

4.2. Existence of Solutions to $(\tilde{S})$ VIA D.c. Problems

For $x \in X$, consider the following parameterized d.c. problem

$$
\mathcal{D C P}(x): \quad \operatorname{Min}_{y \in \mathbb{R}^{q}}\left(\hat{F}_{x}-f_{x}\right)(y) .
$$

Proposition 4.2. Assume that the following assumption is satisfied

(4.2) for any $x \in X$, there exists $\bar{y} \in Y$ such that

(i) $\bar{y}$ is a local solution to $\mathcal{D C P}(x)$

(ii) $0 \in \partial f_{x}(\bar{y})$.

Then, the property $(\mathcal{P})$ is satisfied.

Proof. Let $x \in X$. Since $\bar{y}$ is a local solution of the d.c. problem $\mathcal{D C P}(x)$, it follows that $\partial f_{x}(\bar{y}) \subset \partial \hat{F}_{x}(\bar{y})$ (see [17]). Hence, by ii) we have $0 \in \partial f_{x}(\bar{y}) \cap \partial \hat{F}_{x}(\bar{y})$, and the result follows from Proposition 4.1.

Then, we have the following results which relate weak bilevel and d.c. problems concerning the existence of solutions.

Let $(\tilde{S})$ be the weak nonlinear bilevel problem considered in Section 3 with the corresponding objective functions $\tilde{f}$ and $\tilde{F}$ (recall that $\tilde{X}$ and $\tilde{Y}$ are respectively a compact and a convex compact sets).

Corollary 4.1. Let assumptions (3.1)-(3.5) hold. If moreover, assumption (4.2) is satisfied by the functions $\tilde{F}$ and $\tilde{f}$, then the problem $(\tilde{S})$ has at least one solution.

Proof. Proposition 4.2 implies that the property $(\mathcal{P})$ is satisfied. Then, the existence of solutions is deduced by application of Theorem 3.2.

Corollary 4.2. Let assumptions (3.1)-(3.5) hold. If moreover, the following assumption is satisfied:

(4.3) For any $x \in \tilde{X}$, there exists $\bar{y} \in \tilde{Y}$, verifying

(i) there exists a neighbourhood $\mathcal{U}_{x}(\bar{y})$ of $\bar{y}$ in $\mathbb{R}^{q}$, such that

$$
\partial \tilde{f}_{x}(y) \cap \partial\left(-\tilde{F}_{x}\right)(\bar{y}) \neq \emptyset \quad \forall y \in \mathcal{U}_{x}(\bar{y})
$$


(ii) $0 \in \partial \tilde{f}_{x}(\bar{y})$

then the problem $(\tilde{S})$ has at least one solution.

Proof. In fact, from Corollary 1, [18], $\bar{y}$ is a local solution of $\left(-\tilde{F}_{x}\right)-\tilde{f}_{x}$ on $\mathbb{R}^{q}$, and hence $\partial \tilde{f}_{x}(\bar{y}) \subset \partial\left(-\tilde{F}_{x}\right)(\bar{y})$. Since $0 \in \partial \tilde{f}_{x}(\bar{y})$ it follows that $0 \in \partial \tilde{f}_{x}(\bar{y}) \cap$ $\partial\left(-\tilde{F}_{x}\right)(\bar{y})$, which completes the proof.

\section{Concluding Remarks And COMments}

In this paper, we have shown that a class of Min Sup problems with linked constraints which satisfy the property $(\mathcal{P})$ admit solutions. The obtained result can be applied to the class of weak nonlinear bilevel problems considered in Section 3 (i.e. the class of problems which satisfy assumptions (3.1)-(3.6)). Hence, the property $(\mathcal{P})$ gives another alternative for the existence of solutions to weak nonlinear bilevel problems. Furthermore, for such a class, this property (which in this case corresponds to assumption (3.6)) allows us to deduce the existence of solutions from the existence of solutions of appropriate d.c. problems. Therefore, such a property creates the first link in the literature between the classes of weak nonliear bilevel and d.c. problems. However, it is worthwhile to give some comparison about Section 3 with other works and also some comments. In [16], Lucchetti et al. have given some examples for which the considered weak bilevel problems do not have solutions. In the given Examples 2.1, 4.2 and 4.3, the considered problems do not belong to our class of problems considered in Section 3, because the leader's objective function which they denoted by $f$ do not satisfy the condition: for any $x \in X$, the function $f(x,$.$) is concave. However, in Example 4.1, which$ is the Example 3.1 considered in this paper, as we have seen all assumptions are satisfied.

In [14], Loridan and Morgan have given some results for approximate solutions of a weak bilevel problem $(S)$ via a theoretical approximation scheme. The approximate problems $\left(S_{n}\right)$ correspond to the case where the leader's objective function $f_{1}$, and the follower's objective function $f_{2}$ are respectively replaced by $f_{1, n}$ and $f_{2, n}$. The main result that they obtain concerns only the $\epsilon$-approximate solutions of $(S)$. More precisely, for $\epsilon>0$, let $M_{1}(\epsilon)$ and $M_{1, n}(\epsilon)$ denote the sets of $\epsilon$-approximate solutions of $(S)$ and $\left(S_{n}\right)$ respectively. Under sufficient conditions, the authors showed that any accumulation point of a sequence of $\epsilon$-approximate solutions of $\left(S_{n}\right)$ is an $\epsilon$-approximate solution of $(S)$, that is

$$
\operatorname{Limsup}_{n \rightarrow+\infty} M_{1, n}(\epsilon) \subset M_{1}(\epsilon) .
$$

An interesting question is: what can be said when $\epsilon \rightarrow 0^{+}$?

The theoretical approximation scheme that we have presented in Section 3, differs from the one given in [14], in the fact that it is generated when we substitute the set of solutions $\mathcal{M}(x)$ (for an announced strategy $x$ by the leader) by the set of $\epsilon_{n}$-approximate solutions of the follower $\left(\epsilon_{n} \searrow 0^{+}\right)$. Furthermore, the main result 
that we have given under sufficient conditions concerns directly the existence of solutions to the problem $(S)$.

Acknowledgements. The authtors would like to thank an anonymous referee for his precise remarks and valuable suggestions.

\section{REFERENCES}

[1] A. Aboussoror and P. Loridan, Existence of Solutions to Two-Level Optimization Problems with Nonunique Lower-Level Solutions. J. Math. Anal. Appl. 254 (2001) 348-357.

[2] A. Aboussoror, Weak Bilevel Programming Problems: Existence of Solutions. Adv. Math. Res. 1 (2002) 83-92.

[3] A. Aboussoror and A. Mansouri, Weak linear bilevel programming problems: existence of solutions via a penalty method. J. Mat. Anal. Appl. 304 (2005) 399-408.

[4] A. Aboussoror and A. Mansouri, Sufficient conditions for Min Sup problems and application to bilevel programs, in Proc CIRO'05, IV Conférence Internationale en Recherche Opérationnelle, Théorie et Applications 1 (2005) 99-107.

[5] J.-P. Aubin and I. Ekeland, Applied Nonlinear Analysis. Pure and Applied Mathematics (New York), John Wiley \& Sons, New York (1984).

[6] H. Attouch, Variational Convergences for Functions and Operators. Pitman, Boston (1984).

[7] B. Bank, J. Guddat, D. Klatte, B. Kummer and K. Tammer, Non-Linear Parametric Optimization. Akademie-Verlag, Berlin (1982).

[8] E. De Giorgi and T. Franzoni, Su un Tipo di Convergenza Variazionale. Atti Accad. Naz. Lincei Sci. Fi. Mat. Natur. 58 (1975) 842-850.

[9] S. Dempe, Annotated bibliography on bilevel programming and mathematical programs with equilibrium constraints. Optimization 52 (2003) 333-359.

[10] A.L. Dontchev and Zolezzi, Well-Posed Optimization Problems. Lect. Notes in Mathematics. Springer-Verlag, Berlin. 1543 (1993).

[11] J.E. Falk, A linear Max-Min problem. Math. Program. 5 (1973) 169-188.

[12] M.B. Lignola and J. Morgan, Topological existence and stability for Min-Sup problems. J. Math. Anal. Appl. 151 (1990) 164-180.

[13] M.B. Lignola and J. Morgan, Semicontinuities of marginal functions in a sequential setting. Optimization 24 (1994) 241-252.

[14] P. Loridan and J. Morgan, Approximate Solutions for Two-Level Optimization Problems, in Trends in Mathematical Optimization, International Series of Numerical Mathematics, edited by K. Hoffman, J.-B. Hiriart-Urruty, C. Lemarechal and J. Zowe, Birkhäuser Verlag, Basel 84 (1988) 181-196.

[15] P. Loridan and J. Morgan, On Strict $\epsilon$-Solutions for Two-Level Optimization Problem, in Operations Research Proceedings of the International Conference on Operations Research 90, Vienna, edited by W. Buhler, G. Feichtinger, F. Hartl, F.J. Radermacher and P. Stahly, Springer-Verlag, Berlin (1992) 165-172.

[16] R. Lucchetti, F. Mignanego and G. Pieri, Existence theorem of equilibrium points in Stackelberg games with constraints. Optimization 18 (1987) 857-866.

[17] C. Michelot, Caractérisation des minima locaux des fonctions de la classe D.C., Technical Note, University of Dijon (1987).

[18] Pham Dinh Tao and Le Thi Hoai An, Convex analysis approach to d.c. programming: theory, algorithms and applications. Acta Mathematica Vietnamica 22 (1997) 289-355.

[19] R.T. Rockafellar, Convex analysis. Princeton University Press, Princeton, NJ (1970).

[20] K. Shimizu and E. Aiyoshi, Necessary Conditions for Min-Max Problems and algorithms by a relaxation procedure. IEEE Transactions on Automatic Control: AC-25(1) (1980) 62-66. 